\section{Letter to the Editor Reply to "Re: Aggressive Surgical Approach for Patients with T4 Gastric Carcinoma: Promise or Myth?"}

We are pleased to respond Song et al.'s comments of our article "Aggressive Surgical Approach for Patients with T4 Gastric Carcinoma: Promise or Myth" published in January issue of Annals of Surgical Oncology. In this article, we strictly adhered to the pathological classification of gastric cancer based on AJCC, sixth edition. Of 179 patients with cT4 gastric carcinoma, there were 57 cT4 (pT3) with multiorgan resection (MOR), 91 pT4 with MOR, and $31 \mathrm{cT} 4$ without MOR. Furthermore, among 91 pT4 with MOR, 63\% (58/91) had two or more organs resected, in addition to gastrectomy, whereas only 18 patients (19\%) had pathologically proven cancer cell infiltrations in all resected organs. Nevertheless, we emphasize that all of these 91 pT4 with MOR had, at least, one adjacent organ substantially infiltrated by the cancer cells proven pathologically.

Our data demonstrated that gastrectomy with MOR was associated with considerable morbidity rates $(28.8 \%)$ even in a high-volume surgical center, whereas this surgical approach only led a suboptimal long-term survival: 22.4 and $12.2 \%$ of 3 - and 5 year overall survival, respectively. We totally agree Song et al.'s opinion that advanced nodal involvement is inevitably associated with advanced disease status of the main mass lesion of the stomach, and vice versa. We are not going to discourage aggressive surgical approach, such as pancreatectomy, because the pancreas was the most frequently invaded and resected organ (59\%) in the present series; nevertheless our data showed that 3 year overall survival was extremely dismal $(9.7 \%)$ whenever the pancreas was pathologically invaded. Again, the 3 year survival was even worse $(5.3 \%)$ for N3 disease, which is why we currently do not advocate a liberal surgical attitude to deal with advanced nodal disease and pancreatic involvement. From this point of view, an effective neoadjuvant and/or adjuvant chemo- and/or target therapy for gastric carcinoma is eagerly warranted, which might make the aggressive surgical work more merited.

\section{Ta-Sen Yeh, MD, PhD}

Department of Surgery, Chang Gung Memorial Hospital, Chang Gung University, Taipei, Taiwan

e-mail: tsy471027@adm.cgmh.org.tw

Published Online: 2 July 2011

(C) Society of Surgical Oncology 2011 